\title{
Contiguous papillary thyroid cancer and mediastinal lymphoma
}

\author{
Bell AM ${ }^{1}$, Crothers BA ${ }^{2}$, McManamon AC ${ }^{3}$, Stocker DJ ${ }^{4}$, Burch HB ${ }^{1}$ \\ 1. Endocrine-Metabolic Service, Department of Medicine, Walter Reed National Military Medical Center; Uniformed \\ Services University of the Health Sciences, Bethesda, Maryland, United States. 2. Cytology Section, Department of \\ Pathology, Walter Reed National Military Medical Center, Bethesda, Maryland, United States. 3. Hematology-Oncology \\ Service, Department of Medicine, Wright-Patterson Medical Center, Wright-Patterson AFB, United States. 4. Nuclear \\ Medicine Service, Department of Radiology, Walter Reed National Military Medical Center, Bethesda, Maryland, United \\ States.
}

Correspondence: Henry B. Burch. Address: Endocrine-Metabolic Service, Department of Medicine, Walter Reed National Military Medical Center, Bethesda, Maryland 20889, United States. E-mail: Henry.Burch@us.army.mil

Received: February 10, 2014

Accepted: April 22, 2014

Online Published: April 28, 2014

DOI : $10.5430 /$ crcp.v1n2p47

URL: http://dx.doi.org/10.5430/crcp.v1n2p47

\begin{abstract}
Patients with differentiated thyroid cancer and extensive local invasion have a generally poor prognosis. We present a patient with a large papillary thyroid cancer and apparent substernal extension with aggressive local invasion. Sternotomy with an intent to debulk the tumor revealed inoperable disease, but open biopsy unexpectedly revealed a large B-cell mediastinal lymphoma completely enveloping the great vessels and bronchial tree and juxtaposed to a large but noninvasive papillary thyroid cancer. This finding allowed proper curative therapy for what otherwise might have been considered inoperable thyroid cancer with anaplastic transformation. Seven years following diagnosis the patient remains disease free from both the thyroid cancer and mediastinal lymphoma.
\end{abstract}

\section{Key words}

Thyroid cancer, Mediastinal lymphoma, Diagnosis, Therapy

\section{I ntroduction}

While most patients with thyroid cancer enjoy an excellent prognosis ${ }^{[1]}$, those with aggressive local tumor extension have diminished long term survival generally with death occurring as a result of asphyxia, vascular compromise, or associated distant metastases ${ }^{[2,3]}$. To reflect this poor prognosis, the American Joint Committee on Cancer has reclassified patients greater than 45 years of age with thyroid cancer and extensive local invasion as having stage IV disease ${ }^{[4]}$. The current report describes a patient with a large papillary thyroid cancer and apparent substernal extension with aggressive local invasion. Sternotomy followed by open biopsy revealed a large B-cell mediastinal lymphoma completely enveloping the great vessels and bronchial tree and juxtaposed to a large but noninvasive papillary thyroid cancer. This unexpected finding allowed proper curative therapy for what otherwise might have been considered inoperable thyroid cancer with anaplastic transformation. A dearth of similar cases in the literature and a cautionary note regarding coincident diagnoses makes this case of some interest to health care providers dealing with thyroid cancer. 


\section{Patient and methods}

\subsection{History}

A 42-year-old male sought medical attention in November 2006 with complaints of fever, cough, and hoarseness. The patient was a nonsmoker, and other than a history of hypertension on monotherapy, had been in good health. He was diagnosed with bronchitis and prescribed a brief course of oral antibiotics. A goiter was noted at that visit and a thyroid ultrasound showed a $3.8 \mathrm{~cm}$ right lower pole thyroid nodule. The patient was referred to an otolaryngology clinic where direct laryngoscopy revealed left true vocal cord paralysis. Fine needle aspiration (FNA) was insufficient for analysis and the patient was scheduled for thyroidectomy. A preoperative chest radiography showed an anterior mediastinal mass (see Figure 1 A, B), and the patient was referred to the Endocrinology Service at Walter Reed Army Medical Center for further evaluation. On examination, the patient was a healthy-appearing male in no apparent distress. Neck palpation revealed a mobile and non-tender right lower pole thyroid nodule without associated adenopathy. Lungs were clear to auscultation, and abdominal examination was unremarkable.

Figure 1. The PA (A) and lateral (B) views of the CXR demonstrate a widened mediastinum with tracheal deviation to the right and a soft-tissue density consistent with an anterior mediastinal mass. An infiltrate is also seen in the upper lobe of the left lung.

(C) Transaxial CT images of the neck and upper chest demonstrate calcifications within the right thyroid lobe (arrow) and superior and anterior mediastinal adenopathy as well as a consolidation in the upper lobe of the left lung (D).

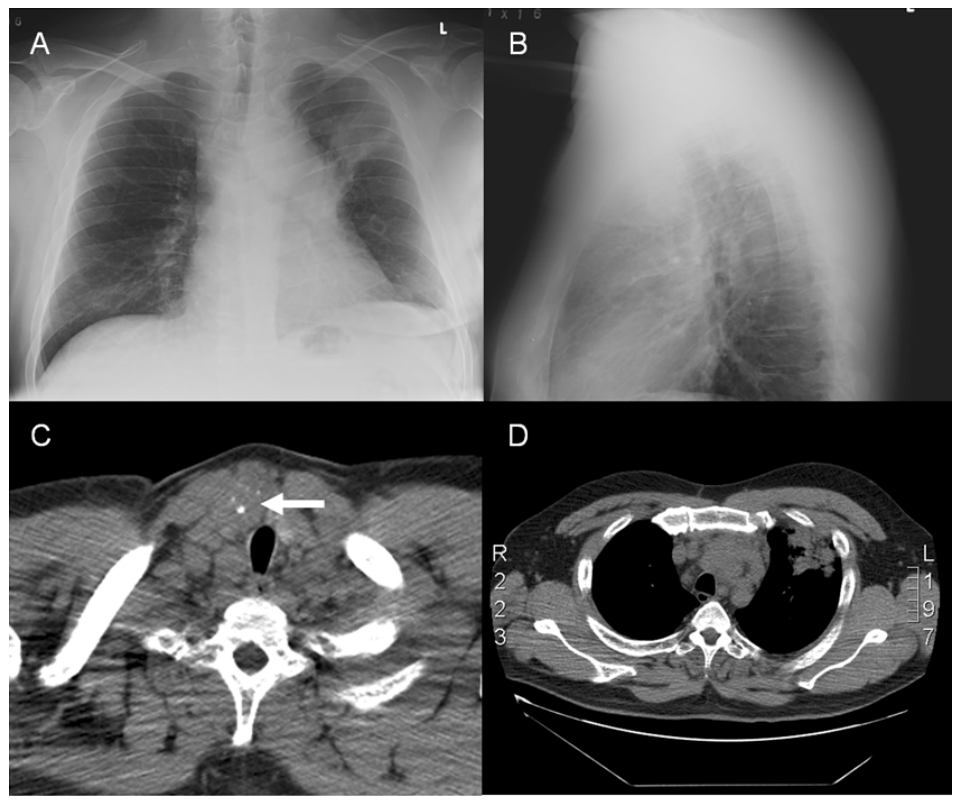

Serum chemistry was normal. TSH was $2.06 \mathrm{mIU} / \mathrm{L}$, free T4 $1.050 \mathrm{ng} / \mathrm{dl}$ (normal 1.01-1.79 ng/dl), serum calcitonin 2.0 $\mathrm{pg} / \mathrm{ml}$, and serum thyroglobulin was $62.9 \mathrm{ng} / \mathrm{ml}$. Anti-thyroid peroxidase and anti-thyroglobulin antibodies were negative. Repeat thyroid FNA in the endocrinology clinic revealed cytological features consistent with papillary thyroid cancer.

Computed tomography of the neck and chest showed a $2.7 \mathrm{~cm}$ right paratracheal (thyroid) mass with punctate calcifications, enlargement of mediastinal lymph nodes, the largest of which was $2.2 \mathrm{~cm}$, and a large anterior mediastinal mass ventral to the aortic arch and pulmonary artery and measuring $10.8 \mathrm{~cm} \times 5.3 \mathrm{~cm}$ (see Figure $1 \mathrm{C}$, D). Within the left upper lung field there was a region of airspace consolidation noted. MRI of the chest showed a large infiltrative $6.5 \mathrm{~cm}$ long $\times 11.4 \mathrm{~cm}$ AP $\times 9.8 \mathrm{~cm}$ wide mass extending downward from the region of the thyroid bed inferiorly to the prevascular space and into the aortopulmonary window (see Figure 2 A, B). The mediastinal mass appeared to invaginate between the aorta and pulmonary artery and wrap around the anterior margin of the main pulmonary artery and aorta. No fat plane was seen between this mass and the great vessels. Positron emission tomography and computed tomography (PET-CT) imaging of the lower neck and chest demonstrated a large hypermetabolic soft tissue mass extending from the region of the thyroid into the anterior mediastinum (see Figure 2 C, D shows PET images only). Incorporated into this mass was a mantle of enlarged, hypermetabolic lymph nodes extending from the anterior cervical chain inferiorly to include supraclavicular, substernal, superior and anterior mediastinal, aortic, bilateral hilar, and pericardial nodes. The 
conglomeration measured $12.4 \mathrm{~cm}$ in maximum diameter. There was not a distinct separation in structure or activity between the inferior margin of the thyroid and the anterior mediastinal mass. The maximum standardized uptake values (SUV-m) were 17.4 for the anterior mediastinal mass and 12.3 for the thyroid nodule, respectively.

Figure 2. Coronal (A) and sagittal (B) MRI images of the chest demonstrate a heterogeneous soft tissue mass deviating the trachea to the right (arrow) and extending from the region of the thyroid into the anterior mediastinum without a distinct tissue plane of separation (arrows). A left upper lobe pulmonary consolidation is again appreciated. Sagittal (C) and coronal (D) PET images demonstrate a mantle of hypermetabolic tissue extending from the neck into the anterior mediastinum, corresponding to the soft tissue masses seen on the above anatomic images and on PET-CT fusion images (not shown).

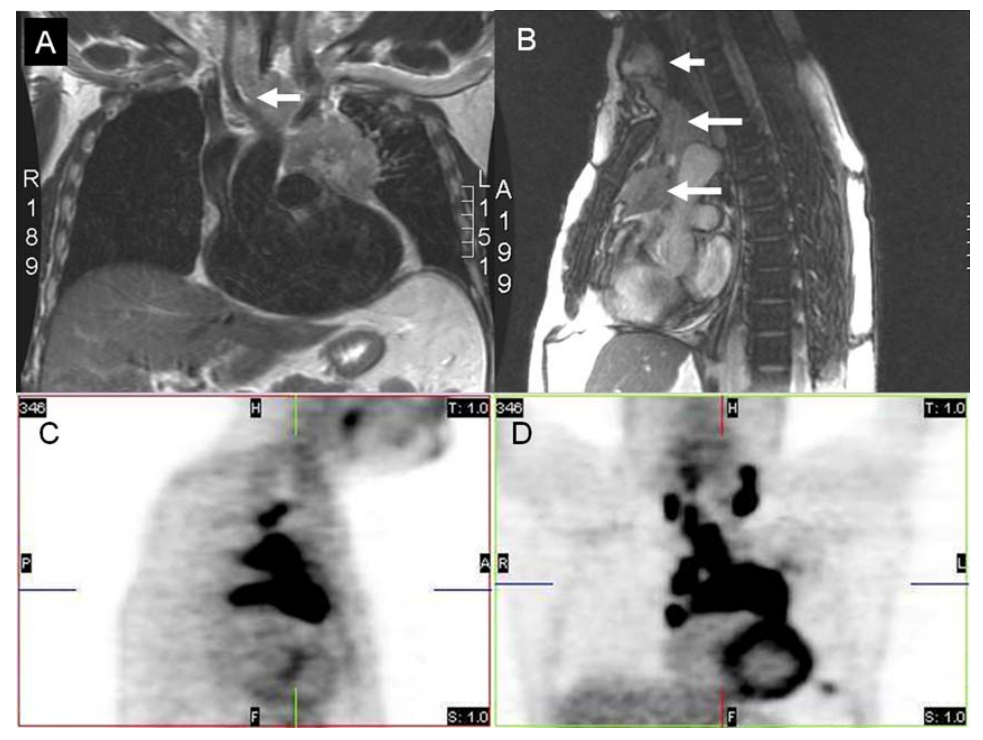

\subsection{Hospital course}

The patient began to experience blood-tinged sputum and was admitted to the inpatient medical service at Walter Reed Army Medical Center. Bronchoscopy was performed, showing no active bleeding and no intraluminal disease. Bronchial alveolar lavage of the left upper lung bronchus was non-diagnostic. The patient underwent CT-guided biopsy of the anterior mediastinal mass, revealing scant atypical cells which were otherwise non-diagnostic. A median sternotomy was performed in an attempt to debulk the mediastinal mass, but the tumor was deemed non-resectable due to extensive involvement of the pulmonary hilum on the left. Open biopsy revealed a diffuse large B cell lymphoma, believed to be of primary mediastinal origin (see Figure 3 A, B). The patient underwent bone marrow aspiration and biopsy with no evidence of bone marrow involvement. CT scan of the abdomen and pelvis also failed to reveal additional evidence of disease.

Figure 3. Diffuse large cell lymphoma of the mediastinum.

A. A large binucleate lymphoid cell with moderate cytoplasm, open vesicular chromatin and prominent nucleolus characterizes the malignancy. Smaller malignant cells are scattered in the background. High power $(600 \times)$, H\&E. B. The mediastinal fat is diffusely infiltrated with large malignant lymphocytes.

Note. Low power $(40 \times)$, H\&E.

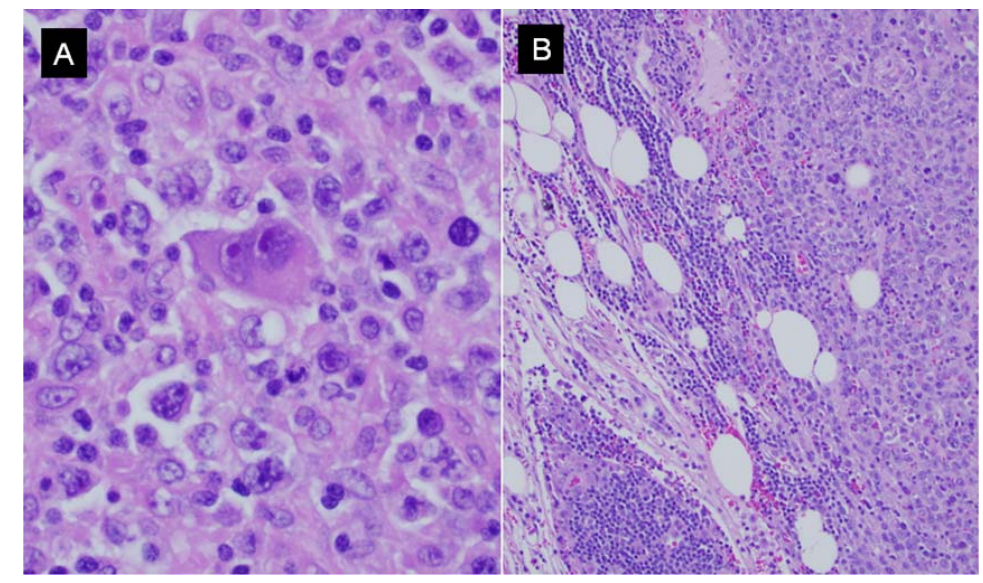

The patient's subsequent care was prioritized as follows. While undergoing preoperative evaluation he was noted to have an abnormal electrocardiogram, which further evaluation showed to be due to a prior inferolateral wall myocardial infarction. On echocardiogram, the patient had moderate inferior and septal wall hypokinesis, and a left ventricular ejection fraction of $40 \%-45 \%$. Subsequent cardiac catheterization revealed total occlusion of the right coronary artery and 
85\% occlusion of the left circumflex artery, for which he received a bare metal stent. He next underwent intensive chemotherapy for his primary mediastinal large B-cell lymphoma (PMBCL), including six cycles of dose-adjusted etoposide, prednisone, vincristine, cyclophosphamide, doxorubicin, and rituximab (DA-EPOCH-R), with a complete response as manifested by disappearance of PET uptake in the mediastinum. Next, the patient underwent a total thyroidectomy without complication, with findings of a $4.0 \mathrm{~cm}$ papillary thyroid cancer with elements of both papillary and follicular differentiation (see Figure 4 A, B). There was no evidence of dedifferentiation and no evidence of primary thyroid lymphoma.

Figure 4. Papillary carcinoma of thyroid.

A. The lesion shows both papillary (right) and follicular (left) architecture. High power (400×), H\&E.

B. Papillary carcinoma of thyroid. Prototypical enlarged, disordered follicular nuclei with cytoplasmic invaginations and nuclear clefts. High power (600×); H\&E.

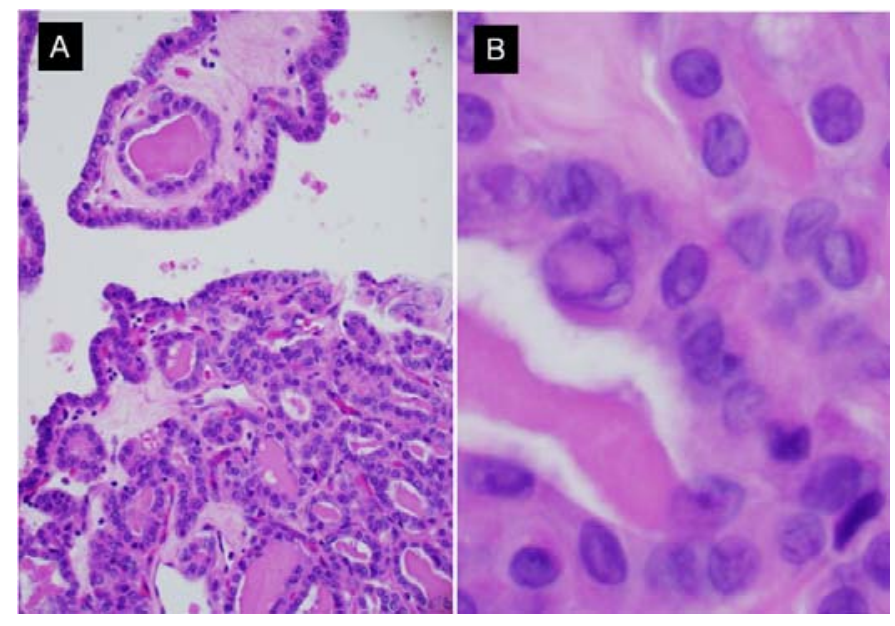

\subsection{Literature search}

A Medline literature search was carried out using the terms thyroid cancer and mediastinal lymphoma, without limitation to years of publication or language. No previous reports of simultaneous thyroid cancer and mediastinal lymphoma were found.

\section{Discussion}

It is estimated that approximately 60,220 new cases of thyroid carcinoma will be diagnosed in the United States in $2013^{[5]}$. Among these, $80 \%$ are stage 1 and stage 2 disease and generally cured with near-total thyroidectomy and radioiodine remnant ablation. Direct extension of papillary thyroid cancer into the soft tissues of the neck occurs in 5\%-15\% of patients with papillary thyroid cancer, and confers a negative prognosis ${ }^{[2]}$. Whereas ten-year mortality for patients with stage 1 or stage 2 disease is less than $5 \%$, nearly one third of patients with extrathyroidal extension will die from their disease by ten years from the time of diagnosis. In a review by McConahey and colleagues, the cause of death from papillary thyroid cancer was aggressive local invasion in 36\% of cases and distant metastases in $39 \%$ of cases ${ }^{[6]}$. Predictors of mortality include invasion of the trachea or esophagus and incomplete surgical excision ${ }^{[2]}$.

Mediastinal involvement with thyroid cancer can occur as a result of either metastatic disease to mediastinal lymph nodes or by direct tumor extension from the thyroid bed. Whereas substernal extension of nodular goiter is a relatively common occurrence, direct substernal extension of thyroid cancer is rare. Torre and colleagues reported on 30 cases of mediastinal extension of primary thyroid cancer among 4,688 patients undergoing thyroidectomy for both benign and malignant thyroid disease ${ }^{[7]}$. Among the 30 patients with mediastinal extension, 14 (47\%) had papillary thyroid cancer, seven had follicular thyroid cancer, five medullary thyroid cancer, and four undifferentiated thyroid cancer. The average age of these patients was 67 years, and 10 -year survival was only $26 \%{ }^{[7]}$.

In patients identified as having an unknown mediastinal mass, the likelihood of malignancy depends upon mass location, patient age, and associated symptoms ${ }^{[8]}$. Anterior mediastinal masses are more likely to be malignant than middle or 
posterior mediastinal masses. In a review of 400 cases of mediastinal masses, 59\% of anterior mediastinal masses were malignant, compared to $29 \%$ and $16 \%$ of middle and posterior mediastinal masses, respectively ${ }^{[9]}$. Patients with malignant tumors presented with local symptoms such as cough, chest pain, and dyspnea and other local symptoms $85 \%$ of the time compared to $46 \%$ in patients with benign masses. The peak age at presentation for patients with mediastinal lymphomas and germinomas is in the second to fourth decades. Most primary mediastinal lymphomas originate in the thymus. Patients described in the literature tend to be young, with bulky disease that is most often confined to the thorax and contiguous structures ${ }^{[10]}$. Most patients have an excellent clinical response to multidrug chemotherapy, with $80 \%$ complete response rates, and $60 \%-80 \%$ relapse-free survival at five years ${ }^{[10-13]}$. In the present case, the temporal association between the discovery of the macroscopic papillary thyroid cancer and a contiguous mediastinal mass suggested a unifying diagnosis of aggressive, locally invasive thyroid cancer. The imaging studies appeared to confirm this suspicion, showing apparent continuity between the thyroid lesion and the invasive mediastinal mass. Arguing against this possibility were the rarity with which papillary thyroid cancer behaves in this fashion, the fact that the vocal cord paralysis occurred on the contralateral side to the thyroid cancer (most likely due to the patient's lymphoma), and an only modest elevation of serum thyroglobulin. An attempt at percutaneous tissue biopsy failed, leading ultimately to a sternotomy with open biopsy revealing simultaneous thyroid cancer and primary mediastinal large B-cell lymphoma. Failure to make this determination would have led to the conclusion that the patient had inoperable widely invasive thyroid cancer, which would have portended a more dismal prognosis. Likewise the patient's lymphoma would not have received appropriate therapy had this distinction not have been made. The key learning point illustrated by the current case is that an atypical presentation of thyroid cancer should lead to a consideration of alternative diagnoses, which should be pursued aggressively if an appropriate therapeutic regimen is to be delivered.

\section{Disclosure statement}

The views expressed in this manuscript are those of the authors and do not reflect the official policy of the Department of the Army, Air Force, the Department of Defense or the United States Government. One or more of the authors are military service members (or employee of the U.S. Government). This work was prepared as part of our official duties. Title 17 U.S.C. 105 provides the "Copyright protection under this title is not available for any work of the United States Government”. Title 17 U.S.C. 101 defines a U.S. Government work as a work prepared by a military service member or employee of the U.S. Government as part of that person's official duties. We certify that all individuals who qualify as authors have been listed; each has participated in the conception and design of this work, the analysis of data (when applicable), the writing of the document, and/or the approval of the submission of this version; that the document represents valid work; that if we used information derived from another source, we obtained all necessary approvals to use it and made appropriate acknowledgements in the document; and that each takes public responsibility for it.

\section{References}

[1] Mazzaferri EL. Management of low-risk differentiated thyroid cancer. Endocr Pract. 2007; 13(5): 498-512. PMid: 17872353. http://dx.doi.org/10.4158/EP.13.5.498

[2] McCaffrey TV, Bergstralh EJ, Hay ID. Locally invasive papillary thyroid carcinoma: 1940-1990. Head Neck. 1994; 16(2): 165-72. PMid: 8021137. http://dx.doi.org/10.1002/hed.2880160211

[3] Ito Y, Miyauchi A, Jikuzono T, et al. Risk factors contributing to a poor prognosis of papillary thyroid carcinoma: validity of UICC/AJCC TNM classification and stage grouping. World J Surg. 2007; 31(4): 838-48. PMid: 17347900. http://dx.doi.org/10.1007/s00268-006-0455-0

[4] Greene FL PD, Fleming ID, et al. AJCC Cancer Staging Manual, 6th ed. New York: Springer-Verlag; 2002. http://dx.doi.org/10.1007/978-1-4757-3656-4

[5] Siegel R, Naishadham D, Jemal A. Cancer statistics. CA Cancer J Clin. 2013; 63: 11-30. PMid: 23335087. http://dx.doi.org/10.3322/caac.21166

[6] McConahey WM, Hay ID, Woolner LB, van Heerden JA, Taylor WF. Papillary thyroid cancer treated at the Mayo Clinic, 1946 through 1970: initial manifestations, pathologic findings, therapy, and outcome. Mayo Clin Proc. 1986; 61(12): 978-96. http://dx.doi.org/10.1016/S0025-6196(12)62641-X 
[7] Torre GC, Ansaldo GL, Borgonovo G, et al. Cervico-mediastinal extension of thyroid cancer. Am Surg. 2000; 66(5): 487-90. PMid: 10824751.

[8] Duwe BV, Sterman DH, Musani AI. Tumors of the mediastinum. Chest. 2005; 128(4): 2893-909. PMid: 16236967. http://dx.doi.org/10.1378/chest.128.4.2893

[9] Davis RD, Jr., Oldham HN, Jr., Sabiston DC, Jr. Primary cysts and neoplasms of the mediastinum: recent changes in clinical presentation, methods of diagnosis, management, and results. Ann Thorac Surg. 1987; 44(3): 229-37. http://dx.doi.org/10.1016/S0003-4975(10)62059-0

[10] Kirn D, Mauch P, Shaffer K, et al. Large-cell and immunoblastic lymphoma of the mediastinum: prognostic features and treatment outcome in 57 patients. J Clin Oncol. 1993; 11(7): 1336-43. PMid: 8315431.

[11] Savage KJ. Primary Mediastinal Large B-Cell Lymphoma. Oncologist. 2006; 11: 488-495. PMid: 16720849. http://dx.doi.org/10.1634/theoncologist.11-5-488

[12] Wilson WH, Grossbard ML, Stefania Pittaluga, et al. Dose-adjusted EPOCH chemotherapy for untreated large B-cell lymphomas: a pharmacodynamic approach with high efficacy. Blood. 2002; 99: 2685-3. PMid: 11929754.

http://dx.doi.org/10.1182/blood.V99.8.2685 